\title{
An OVR-SVM Based Machine Vision Evaluation Method for Standard Component Assembly
}

\author{
Jian Huang ${ }^{\mathrm{a}}$, Ping Jia ${ }^{\mathrm{b}}$ and Guixiong Liuc, ${ }^{\mathrm{*}}$ \\ School of Mechanical \& Automotive Engineering, South China University of Technology. \\ Guangzhou 510641, China \\ aMEhuangjian@mail.scut.edu.cn, b532289123@qq.com, cMEgxliu@scut.edu.cn
}

\begin{abstract}
Keywords: Standard Component Assembly; Evaluation Method; Machine Vision; One versus Rest.
\end{abstract}
\begin{abstract}
In view of the current evaluation of the assembly quality of the standard components, the artificial visual method is used to judge it. However, when people do it repeatedly and in a highintensity, which is difficult to meet the needs of large-scale industrial production. In this paper, according to production process, the OVR-SVM machine vision method for the assembly quality of standard components is proposed. First, it analyzes the evaluation of assembly quality of standard components and puts forward evaluation method of standard components assembly. Secondly, it uses the One Versus Rest (OVR) strategy to form two sets including correct and incorrect assembly, which can evaluate the quality of assembly standard components by only twice judging. Finally, it finishes the assembly quality evaluation of standard components for the machine testing based on SVM. Experimental results show, in this process, the accuracy of SVM classifier based on OVR strategy is $100 \%$, and it has the characteristics of generalization ability of learning unknown sample, which can meet the needs of testing the assembly quality of the standard components in highly automated assembly line.
\end{abstract}

\section{Introduction}

In the machinery equipment, mold forming, transportation equipment, electronic equipment, data communications equipment, information technology equipment and other manufacturing, the standard components are widely used[1]. Standard components have many characteristics, such as large number of components, complex types and different structures, which is easy to appear misplaced assembly, missing assembly, bad assembly or other incorrect assembly in the process, testing the quality of standard components assembly is an important role in the production[2]. In view of the current evaluation of the assembly quality of the standard components, the artificial visual method is used to judge it. However, when people do it in a high-intensity and repeatedly, which is difficult to meet the needs of large-scale industrial production[3]. Using machines to evaluate the assembly quality to take place of people is an inevitable trend of upgrading intelligent manufacturing industry which improves the quality of products and efficiency of production.

Pattern recognition method of image classification which is based on the observation and learning of known image samples, then finish the classification detection for the new image sample with significant images' features, which is an effective analysis tool for evaluating the assembly quality of cases[5]. At present, the image classification methods in the field of machine vision mainly include k-nearest neighbor algorithm and support vector machine. Treesa George (2014) used the k-NN algorithm to classify and identify still images, reaching an accuracy rate of 66.6\%[6]. The author's team (2015) studied the status of the device status lights based on the k-NN algorithm reaching an accuracy rate of 100\%[7] and (2016) used k-NN classification algorithm to convert the color model and classify and identify them reaching an accuracy rate of 98.65\%[8]. S Begum (2016) Initially three feature selection algorithm CBFS, FPRS and KFRS is applied on the dataset and then k-NN is applied as a classifier onto the dataset[9]. Article [10] (2015) proposed a method to realize the product quality detection in industrial process by using image statistical modeling based on the principle of SVM, and use it on the product quality intelligent inspection line, obtains a high classification accuracy rate. FB Marin (2016) presented the automatic image analysis algorithm and semiautomatic method to 
measure grains in microstructure images, improves performance of the work[11]. Article [12] (2017) used SVM to classify the images and the experimental results show that the SVM algorithm has a high classification accuracy and high reliability.

\section{OVR Based Standard Components Assembly Evaluate Method}

\subsection{Standard Components Assembly Quality Evaluate Needs}

Assemble means of the process of combining components according to the technical requirements, debugging, testing to become a qualified product, assembly began in the design. Different products on a variety of different standard components, in the quality inspection process, the assembly of each assembly location test results can be summarized as the correct assembly, the incorrect assembly of have two cases. (1) the correct assembly means that assemble the correct standard components in the right position according to the technical requirements. (2) the incorrect assembly means that assembled standard components in the position where the standard components are incorrect, and their appearances are failure or even without anything.

In the process of assembly, the position where need to assemble standard components is fixed. We can know the assembly is correct or incorrect by judging whether the correct standard components is assembled in the correct position. A case's three-dimensional model and physical map is shown in Figure 1.
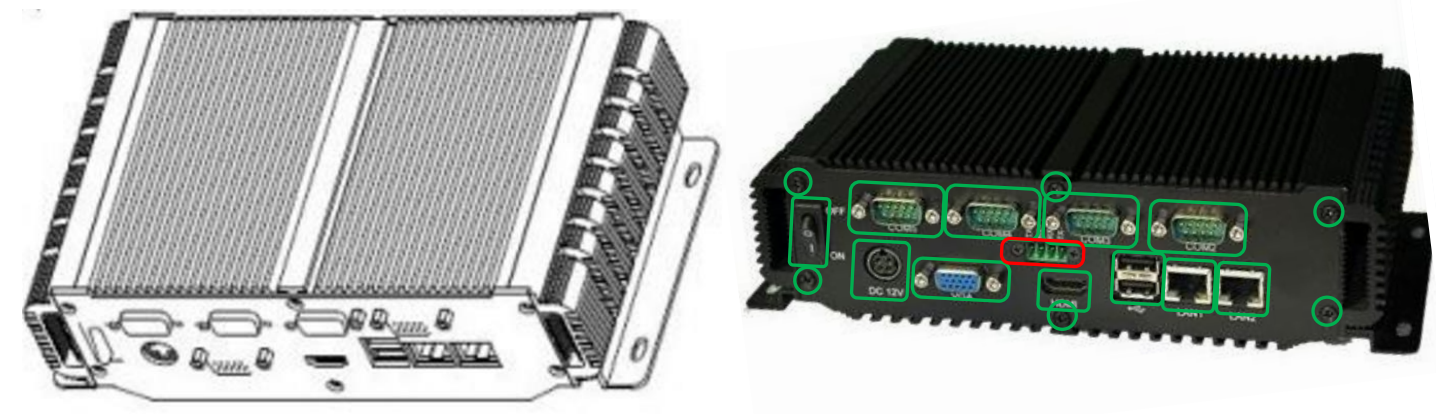

Figure 1. A computer case's three-dimensional model and physical map.

\subsection{Standard Components Assembly Evaluate Method Based on OVR}

If assembled products have $\mathrm{K}$ types of standard components, supposing the case of assembling the number $i$ type of standard component is renamed $\boldsymbol{T}_{i}(i=1,2 \ldots K)$ and the case of assembling nothing is $\boldsymbol{T}_{0}$, all the assembled cases $\mathbb{R}$ can be expressed as follow:

$$
\mathbb{R}=\left\{\boldsymbol{T}_{0}, \boldsymbol{T}_{1}, \boldsymbol{T}_{2}, \cdots, \boldsymbol{T}_{i}, \cdots, \boldsymbol{T}_{K}\right\}(i \in \mathbb{Z}) \quad \boldsymbol{T}_{i}= \begin{cases}\left\{t^{(i)} \mid t^{(i)} \text { is the number } i \text { standard part }\right\} & i>0, i \in \mathbb{Z} \\ \left\{t^{(0)} \mid t^{(0)} \text { is assembling nothing }\right\} & i=0, i \in \mathbb{Z}\end{cases}
$$

Assembly begins with assembly design. Supposing the number i standard component shall be assembled in a certain site while the actual assembly case is renamed $t$, it is correct only if the recognition results are within $\boldsymbol{T}_{i}$, the other cases are incorrect assembly. The sufficient and necessary conditions for the correct or incorrect assembly respectively is shown below.

$$
\left\{t \in \boldsymbol{T}_{i} \Rightarrow \text { correct assembly; } \sum_{j=0, j \neq i}^{K} t \in \boldsymbol{T}_{j} \Rightarrow\right. \text { incorrect assembly }
$$

The correct and the incorrect assembly are mutually exclusive events, but it is necessary to distinguish the correct and the incorrect assembly in order to accurately analyze the assembly quality of standard components, which needs a correct judgment of assembly and $\mathrm{K}$ times of assembly errors. 
In fact, within all assembly cases $\mathbb{R}$, the correct and the incorrect assembly are mutually exclusive events so that One Versus Rest (OVR) strategy is adopted, forming the correct and the incorrect assembly sets, which can evaluate the quality of assembly standard components through only twice correct judgments. For the sites should be assembled the number i standard component, $\mathbb{R}$ can be divided in two opposite sets, the correct assembly set $\mathrm{Ai}$ and the incorrect assembly set $\mathrm{Bi}$, which are as follow:

$$
\boldsymbol{A}_{i}=\left\{t \mid t \in \boldsymbol{T}_{i}, t \in \mathbb{R}\right\}=\boldsymbol{T}_{i} \quad \boldsymbol{B}_{i}=\overline{\boldsymbol{A}}_{i}=\left\{t \mid t \notin \boldsymbol{T}_{i}, t \in \mathbb{R}\right\}=\mathbb{R}-\boldsymbol{T}_{i}=\bigcup_{j=0, j \neq i}^{K} \boldsymbol{T}_{j}
$$

The assemble evaluate equation of each part is shown below.

$$
t \in \boldsymbol{A}_{i} \Rightarrow \text { correct assembly, } t \in \boldsymbol{B}_{i} \Rightarrow \text { incorrect assembly }
$$

Equation (3) and (4) are standard components assembly evaluation technology based on OVR. Figure 2 is the schematic diagram of operation of standard components correct assembly evaluation technology based on OVR. Figure 3 is the sketch map of the correct assembly set and the incorrect assembly set of the number i standard component, using the correct assembly evaluation technology based on OVR. If the recognized standard component type $\mathrm{t}$ is within $\mathrm{Ai}$ (the white area on the right), it is correct assembly. If the recognized standard component type $\mathrm{t}$ is within $\mathrm{Bi}$ (the gray area on the right), it is correct assembly.

\begin{tabular}{|l|l|l|l|l|l|l|}
\hline$T_{0}$ & $T_{1}$ & $T_{2}$ & $\cdots \cdots$ & $T_{i}$ & $\cdots \cdots$ & $T_{k}$ \\
\hline
\end{tabular}

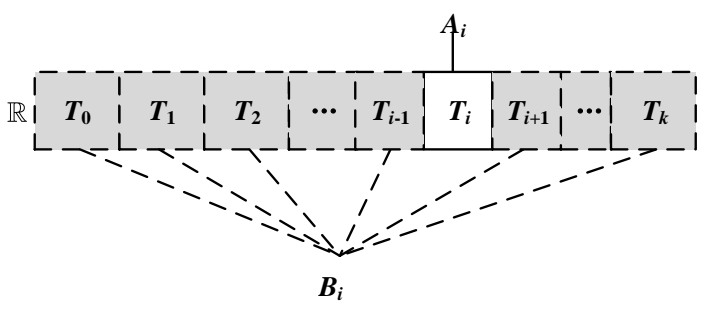

Figure 2. The schematic diagram of operation of standard components correct assembly evaluation technology based on OVR

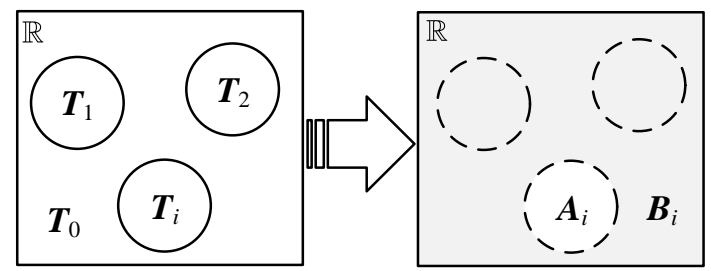

Figure 3. The sketch map of the correct assembly set and the incorrect assembly set of the number i standard component

Example. A assemble station has 5 positions which need to evaluate assemble quality, contains stereo mini $\boldsymbol{T}_{1}, \mathrm{RS} 232$ Serial Interface $\boldsymbol{T}_{2}, \mathrm{RJ} 45 \mathrm{jack} \boldsymbol{T}_{3}$, bolt connector $\boldsymbol{T}_{4}$, camera interface $\boldsymbol{T}_{5}$, and defined $\boldsymbol{T}_{0}$ as non-assemble.

When evaluate the stereo mini assemble quality, according to equation (3) and (4), its correct assemble set $\boldsymbol{A}_{1}=\boldsymbol{T}_{1}$, incorrect set $\boldsymbol{B}_{i}=\overline{\boldsymbol{A}}_{i}=\mathbb{R}-\boldsymbol{T}_{1}=\boldsymbol{T}_{0} \cup \boldsymbol{T}_{2} \cup \boldsymbol{T}_{3} \cup \boldsymbol{T}_{4} \cup \boldsymbol{T}_{5}$. Using OVR evaluate method to divide the correct and incorrect assembly set for each standard components are shown in Table 1

Table 1.Standard components evaluation set by OVR evaluation method

\begin{tabular}{ccc}
\hline Standard Component Type & Correct Assembly Set Ai & Incorrect Assembly Set Bi \\
\hline Stereo Mini & $\boldsymbol{T}_{1}$ & $\boldsymbol{T}_{0} \cup \boldsymbol{T}_{2} \cup \boldsymbol{T}_{3} \cup \boldsymbol{T}_{4} \cup \boldsymbol{T}_{5}$ \\
RS232 Serial Interface & $\boldsymbol{T}_{2}$ & $\boldsymbol{T}_{0} \cup \boldsymbol{T}_{1} \cup \boldsymbol{T}_{3} \cup \boldsymbol{T}_{4} \cup \boldsymbol{T}_{5}$ \\
RJ45 Jack & $\boldsymbol{T}_{3}$ & $\boldsymbol{T}_{0} \cup \boldsymbol{T}_{1} \cup \boldsymbol{T}_{2} \cup \boldsymbol{T}_{4} \cup \boldsymbol{T}_{5}$ \\
Bolt Connector & $\boldsymbol{T}_{4}$ & $\boldsymbol{T}_{0} \cup \boldsymbol{T}_{1} \cup \boldsymbol{T}_{2} \cup \boldsymbol{T}_{3} \cup \boldsymbol{T}_{5}$ \\
Camera Interface & $\boldsymbol{T}_{5}$ & $\boldsymbol{T}_{0} \cup \boldsymbol{T}_{1} \cup \boldsymbol{T}_{2} \cup \boldsymbol{T}_{3} \cup \boldsymbol{T}_{4}$ \\
\hline
\end{tabular}




\section{OVR-SVM Machine Vision Evaluation Method for Standard Components Assembly Quality}

The basic idea of Support Vector Machine (SVM) is using the structural risk minimization learning method, to find the optimal classification surface in the sample distribution space to maximize the separation between the positive and negative sample categories. SVM has been widely used because its unique advantages in solving small sample, nonlinear and high dimensional pattern recognition problems by using slack variable or kernel function to classify nonlinear sample data.

There is a finite number of discrete samples in actual standard component samples, which has $\mathrm{N}$ elements. Defined the ith class actual sample set as $\boldsymbol{T}_{i}^{\prime}$, the actual correct sample set as $\boldsymbol{A}_{i}^{\prime}$, the actual incorrect set as $\boldsymbol{B}_{i}^{\prime}$, the nth element as $t_{n}^{(i)}$

$$
\boldsymbol{A}_{i}^{\prime}=\boldsymbol{T}_{i}^{\prime}=\left\{t_{1}^{(i)}, t_{2}^{(i)}, t_{3}^{(i)}, \cdots, t_{n}^{(i)}, \cdots, t_{N}^{(i)}\right\} ; \quad \boldsymbol{B}_{i}^{\prime}=\bigcup_{j=0, j \neq i}^{K} \boldsymbol{T}_{j}^{\prime}
$$

Figure 4 shows the ith class standard components sample set $\boldsymbol{T}_{i}^{\prime}$ mapped in the full set $\mathbb{R}$.

Every red solid point is a standard component sample $t_{n}^{(i)}$. All of the red solid points represent the standard component samples set $\boldsymbol{T}_{i}^{\prime}$, the red circle represents the ith class standard component situation in theory. If the area of $\boldsymbol{T}_{i}^{\prime}$ is more close to $\boldsymbol{T}_{i}$, the standard components assembly quality evaluation method is more accurate.

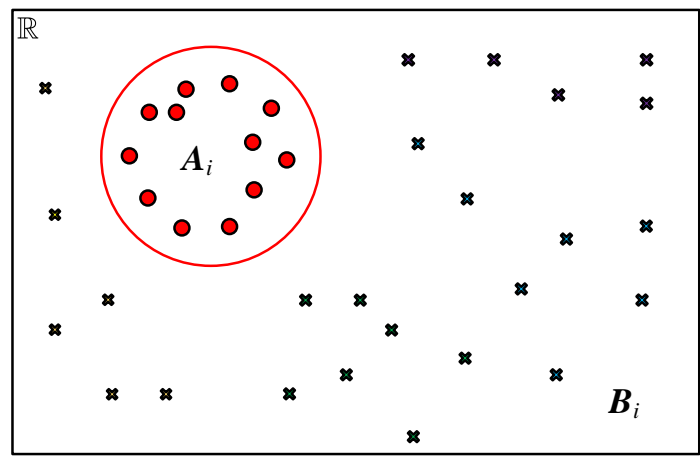

Figure 4. The ith class standard components sample set $\boldsymbol{T}_{i}^{\prime}$ mapped in the full set $\mathbb{R}$

In discrete situation, which the product has $\mathrm{k}$ type standard components to assemble, we can construct k SVM classifiers to evaluate assembly quality. The SVM to evaluate the ith class standard components assembly correct or not is built by the correct assembly set $\boldsymbol{A}_{i}^{\prime}$ and incorrect assembly set $\boldsymbol{B}_{i}^{\prime}$. The learning result of using SVM to some kind of standard component $\boldsymbol{T}_{i}^{\prime}$ is shown in Figure 5. The mapping of learning result of $\boldsymbol{T}_{i}^{\prime}$ in full set $\mathbb{R}$ is like Figure 6, the red solid line is the situation of this type standard components in theory, the blue dashed line is the learning result of this type standard components by SVM, the red dashed line is the mapping of these recognizable standard components in the full set $\mathbb{R}$ by using matching method.
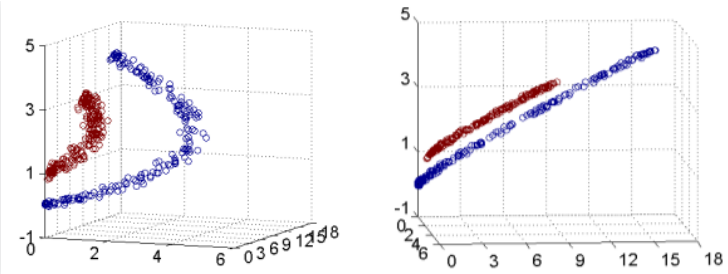

Figure 5. Learning result of using SVM to some kind of standard component $\boldsymbol{T}_{i}^{\prime}$

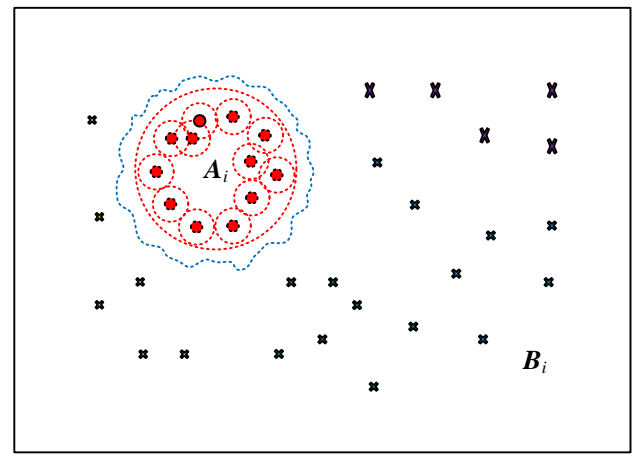

Figure 6. Mapping of learning result of $\boldsymbol{T}_{i}^{\prime}$ in full set $\mathbb{R}$ 
In the evaluation process, the standard parts $\boldsymbol{T}_{i}$ corresponds to a SVM for testing. Evaluation method of OVR-SVM machine vision for standard components assembly quality is shown in Figure 7. After testing started, the system check the first position which should assembled by $\boldsymbol{T}_{i}$, using the corresponding type SVM classifier to judge. Then the SVM classifier will give a judge result to the standard component in this positon. If the result belongs to $\mathrm{Ai}$, then go to the next positon. Otherwise the case is assembled incorrect.

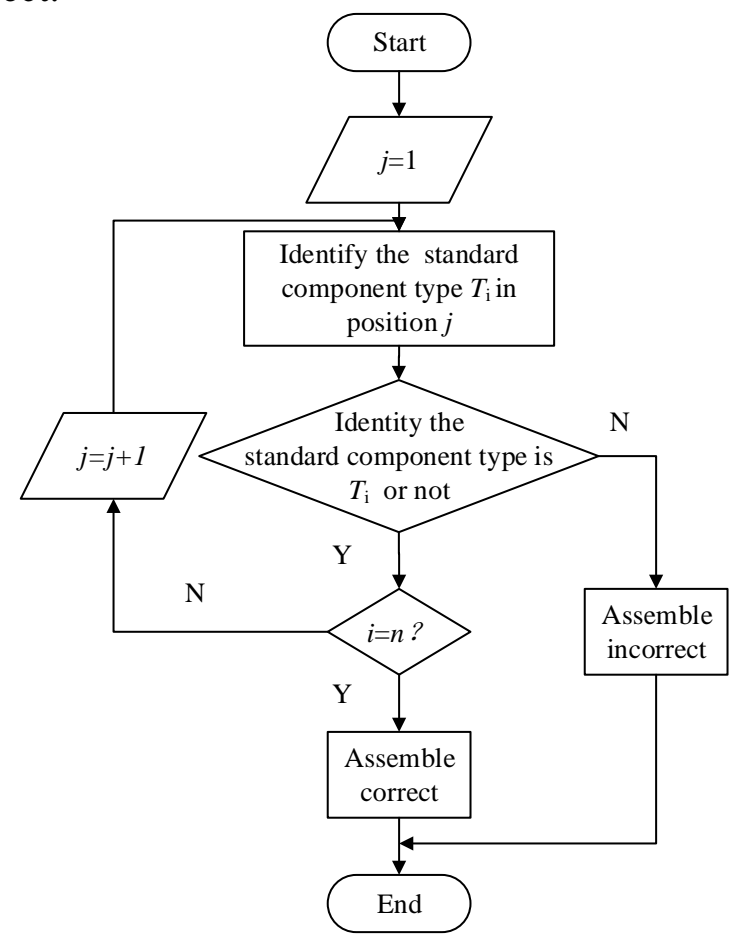

Figure 7. OVR-SVM standard components assembly quality evaluate method flow

Using OVR strategy training multiple SVM classifier to evaluate the machine assembly quality, it is easy to realize and its classify logic is similar to pattern recognition method. The classifier can accept multiple samples to train for a certain case standard components, which has a great generalization ability, can be applied to such as high integration level, lack of flexible automation case assembly production line, ensure the quality of the product assembly qualified, then send to the next role.

\section{Experimental Verification}

Building 3 type standard components image sample set of case functional components, case sheet metal parts and case fasteners for classification evaluation. Firstly, in selecting samples, capture the samples of the standard components of the case under different lighting conditions. Secondly, in consideration of the camera's radial distortion and other image disturbance, collecting standard components image in different position. Meanwhile, the number of samples should be uneven. The 3 type standard components image sample set is built according to the 3 rules above is shown in Table 5-3. Including 204 samples, 5 type case functional components, such as COM interface, DVI interface, PS/2 interface, RJ45 jack and power connecter, 8 type case sheet metal parts, such as PCI fender, case fan sheet metal, power fan sheet metal, power supply interface sheet metal, interface sheet metal, heat exchange net, warning sheet metal sheet metal parts, and case fasteners such as hole rivets and blind rivet.

In classification and matching, select $75 \%$ (153) image samples for training examples (matching template) in random, the other for testing examples (matching target). The OVR structure SVM classification and recognition effect contrast template matching method is shown in Table 3 . In this process of testing, based on the OVR structure, SVM classifier recognition accuracy rate can reach 
$100 \%$ when using different kernel function. It has the characteristics of the unknown sample learning generalization ability.

\section{Conclusion}

This paper presented the evaluation technology of standard components assembly based on OVR, and the test result of the correct or incorrect condition for each assembly location only twice judging.

Based on SVM to realize standard components assembly quality testing machine evaluation, and presents OVR-SVM classifier standard components assemble quality inspection method. The result shows, OVR-SVM classifier recognition accuracy rate reached $100 \%$, have the characteristics of the unknown sample learning generalization ability, can satisfy the highly automated assembly line standard components assembly quality testing requirements.

Table 2. 3 type standard components image sample set of case functional components (Part)

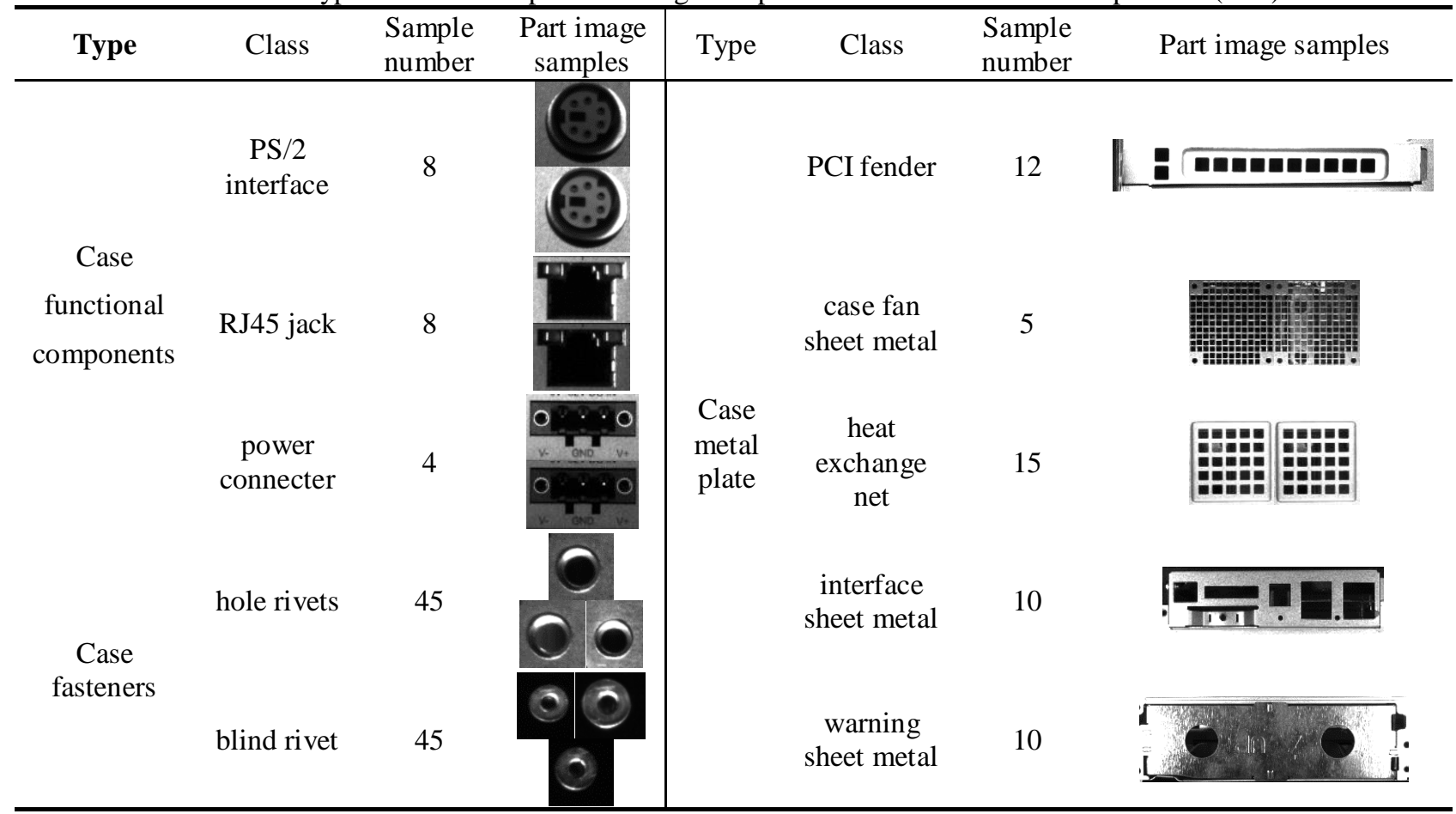

Table 3. OVR structure SVM classification and recognition effect contrast template matching method

\begin{tabular}{|c|c|c|c|c|c|c|c|c|c|}
\hline \multirow[b]{2}{*}{ Type } & \multirow[b]{2}{*}{$\begin{array}{l}\text { Testing } \\
\text { sample } \\
\text { number } \\
\text { (unit/time) }\end{array}$} & \multirow[b]{2}{*}{$\begin{array}{c}\text { Test } \\
\text { times } \\
\text { (Times) }\end{array}$} & \multirow[b]{2}{*}{$\begin{array}{l}\text { Total } \\
\text { test } \\
\text { number } \\
\text { (unit) }\end{array}$} & \multicolumn{3}{|c|}{ Template matching method } & \multicolumn{3}{|c|}{ OVR structure SVM classifier } \\
\hline & & & & $\begin{array}{l}\text { Incorrect } \\
\text { matching } \\
\text { number } \\
\text { (unit) }\end{array}$ & $\begin{array}{l}\text { Correct } \\
\text { matching } \\
\text { number } \\
\text { (unit) }\end{array}$ & $\begin{array}{l}\text { Matching } \\
\text { accuracy } \\
\text { rate }\end{array}$ & $\begin{array}{l}\text { Incorrect } \\
\text { number } \\
\text { (unit) }\end{array}$ & $\begin{array}{l}\text { Correct } \\
\text { number } \\
\text { (unit) }\end{array}$ & $\begin{array}{c}\text { Recognition } \\
\text { accuracy } \\
\text { rate }\end{array}$ \\
\hline $\begin{array}{c}\text { Case } \\
\text { functional } \\
\text { components }\end{array}$ & 11 & 5 & 55 & 43 & 12 & $21.8 \%$ & 0 & 55 & $100.0 \%$ \\
\hline $\begin{array}{l}\text { Case sheet } \\
\text { metal parts }\end{array}$ & 18 & 5 & 90 & 76 & 14 & $15.6 \%$ & 0 & 90 & $100.0 \%$ \\
\hline $\begin{array}{c}\text { Case } \\
\text { fasteners }\end{array}$ & 22 & 5 & 110 & 56 & 54 & $49.1 \%$ & 0 & 110 & $100.0 \%$ \\
\hline Full Set & 51 & 5 & 255 & 175 & 80 & $31.4 \%$ & 0 & 255 & $100.0 \%$ \\
\hline
\end{tabular}

\section{Acknowledgments}

The authors wish to acknowledge the financial support from the Guangzhou Science and Technology Plan Projects (No. 2017010160641 and No. 201509010008). 


\section{References}

[1]. Ibrahim I, Ibrahim Z, Ahmad $\mathrm{H}$, et al. An assembly sequence planning approach with a rulebased multi-state gravitational search algorithm [J]. The International Journal of Advanced Manufacturing Technology, 2015, 79(5):1363-1376.

[2]. Addition I. Modeling of RFID-Enabled Real-Time Manufacturing Execution System in MixedModel Assembly Lines [J]. Mathematical Problems in Engineering, 2015, 2015:1-15.

[3]. Nafisi M, Wiktorsson M, Rösiö C. Manufacturing Involvement in New Product Development: An Explorative Case Study in Heavy Automotive Component Assembly $\hat{\sim}_{[}[\mathrm{J}]$. Procedia Cirp, 2016, 50:65-69.

[4]. Pan W, So K C. Component procurement strategies in decentralized assembly systems under supply uncertainty [J]. IIE Transactions, 2016, 48(3):267-282.

[5]. Li K, Wang F, Zhang L. A new algorithm for image recognition and classification based on improved Bag of Features algorithm [J]. Optik - International Journal for Light and Electron Optics, 2016, 127(11):4736-4740.

[6]. George T, Potty S P, Jose S. Smile detection from still images using K-NN algorithm[C]// International Conference on Control, Instrumentation, Communication and Computational Technologies. 2014:461-465.

[7]. Chen Gengxin, Huang Jian, Liu Guixiong. Pattern recognition based on trusted k-NN for EMC surge test multi state lamps [J]. Journal of electronic measurement and instrumentation, 2015, 29 (11): 1718-1724.

[8]. Guo Xuemei, Liu Guixiong. Color model segmentation by self-learning K-NN device state identification method [J]. China test, 2016, 42 (4): 107-110.Begum S, Chakraborty D, Sarkar R. Data Classification Using Feature Selection and k-NN Machine Learning Approach[C]// International Conference on Computational Intelligence and Communication Networks. IEEE, 2016:811-814.

[9]. Begum S, Chakraborty D, Sarkar R. Data Classification Using Feature Selection and kNN Machine Learning Approach[C]// International Conference on Computational Intelligence and Communication Networks. IEEE, 2016:811-814.

[10]. Liu Jinping, Tang Zhaohui, Zhang Jin. Quality monitoring of industrial process products based on image statistical modeling [J]. opto electronics, lasers, 2015 (9): 1796-1805.

[11]. Marin F B, Potecaşu F, Marin M, et al. Automatic Grain Size Determination in Microstructures Using Computer Vision Algorithm Based on Support Vector Machine (SVM)[J]. Advanced Materials Research, 2017, 1143.

[12]. Xie X, Wang T. A projection twin SVM-based active contour model for image segmentation[C]// International Conference on Mechatronics and Machine Vision in Practice. IEEE, 2017. 